PROCEEDINGS OF THE

AMERICAN MATHEMATICAL SOCIETY

Volume 136, Number 11, November 2008, Pages 4011-4017

S 0002-9939(08)09410-0

Article electronically published on June 2, 2008

\title{
A NOTE ON SCALING ASYMPTOTICS FOR BOHR-SOMMERFELD LAGRANGIAN SUBMANIFOLDS
}

\author{
ROBERTO PAOLETTI
}

(Communicated by Jon G. Wolfson)

\begin{abstract}
This paper deals with the asymptotic expansions describing the quantum states associated to Bohr Sommerfeld Lagrangian submanifolds of a compact Kähler manifold, in the context of geometric quantization. More precisely, it provides an improvement on a result of the work of Debernardi and the author (2006), describing a natural factorization of the expansion and providing certain remainder estimates.
\end{abstract}

\section{INTRODUCTION}

The purpose of this paper is to improve an expansion in [5] for the asymptotics associated to Bohr-Sommerfeld Lagrangian submanifolds of a compact Hodge manifold, in the context of geometric quantization (see e.g. [1, 2 2, 6], 9]). We adopt the general framework for quantizing Bohr-Sommerfeld Lagrangian submanifolds presented in [2, based on applying the Szegö kernel of the quantizing line bundle to certain delta functions concentrated along the submanifold.

Let $M$ be a d-dimensional complex projective manifold, with complex structure $J$; consider an ample line bundle $A$ on it, and let $h$ be a Hermitian metric on $A$ such that the unique compatible connection has curvature $\Omega=-2 i \omega$, where $\omega$ is a Kähler form. Then the unit circle bundle $X \subseteq A^{*}$, endowed with the connection one-form $\alpha$, is a contact manifold. A Bohr-Sommerfeld Lagrangian submanifold of $M$ (or, more precisely, of $(M, A, h)$ ) is then simply a Legendrian submanifold $\Lambda \subseteq X$, conceived as an immersed submanifold of $M$.

In a standard manner, $X$ inherits a Riemannian structure for which the projection $\pi: X \rightarrow M$ is a Riemannian fibration. In view of this, we shall implicitly identify (generalized) functions, densities and half-densities on $X$ at certain places.

Referring to $\S 2$ of [5] for a more complete description of the prelimiaries involved, we recall that if $\Lambda \subseteq X$ is a compact Legendrian submanifold and $\lambda$ is a half-density on it, there is a naturally induced generalized half-density $\delta_{\Lambda, \lambda}$ on $X$ supported on $\Lambda$. Following [2], we can then define a sequence of CR functions

$$
u_{k}=: \mathrm{P}_{k}\left(\delta_{\Lambda, \lambda}\right) \in \mathcal{H}(X)_{k},
$$

Received by the editors October 1, 2007.

2000 Mathematics Subject Classification. Primary 53D12, 53D50; Secondary 81S10, 81Q20, 81Q70.

Key words and phrases. Geometric quantization, Lagrangian submanifolds, asymptotics.

(C)2008 American Mathematical Society Reverts to public domain 28 years from publication 
where $\mathcal{H}(X)_{k}$ is the $k$-th isotype of the Hardy space with respect to the $S^{1}$-action, and $\mathrm{P}_{k}: L^{2}(X) \rightarrow \mathcal{H}(X)_{k}$ is the orthogonal projector (extended to $\mathcal{D}^{\prime}(X) \rightarrow$ $\left.\mathcal{H}(X)_{k}\right)$. In the present setting there are natural unitary structures on $\mathcal{H}(X)_{k}$ and the space of global holomorphic sections $H^{0}\left(M, A^{\otimes k}\right)$, and a natural unitary isomorphism $\mathcal{H}(X)_{k} \cong H^{0}\left(M, A^{\otimes k}\right)$. One thinks of the $u_{k}$ 's as representing the quantizations of $(\Lambda, \lambda)$ at Planck's constant $1 / k$. It is easily seen that $u_{k}$ is rapidly decaying as $k \rightarrow+\infty$ on the complement of $S^{1} \cdot \Lambda=\pi^{-1}(\pi(\Lambda))$; here $\pi: X \rightarrow M$ is the projection. On the other hand, the asymptotic concentration of the $u_{k}$ 's along $S^{1} \cdot \Lambda$ poses an interesting problem, already considered in Theorem 3.12 of [2].

This theme was revisited in [5] in a somewhat different technical setting; in particular, Corollary 1.1 of [5] shows that the scaling asymptotics of $u_{k}$ (to be defined shortly) near any $x \in S^{1} \cdot \Lambda$ admit an asymptotic expansion, and explicitly computes the leading order term. We shall presently give a more precise description of this expansion as a function on the tangent space of $M$ at $m=\pi(x)$. Namely, we shall show that this asymptotic expansion may be factored as an exponentially decaying term in the component $w^{\perp}$ of $w \in T_{m} M$ orthogonal to $\Lambda$ times an asymptotic expansion with polynomial coefficients in $w$ (more precisely, the expansion is generally given by a finite sum of terms of this form, one from each branch of $\Lambda$ projecting to $m$ ); the exponential term also contains a symplectic pairing between $w^{\perp}$ and the component of $w$ along $\Lambda, w^{\|}$. Furthermore, we shall give some relevant remainder estimates not mentioned in [5].

Before stating the results of this paper, let us recall that for any $x \in X$ we can find a Heisenberg local chart for $X$ centered at $x$,

$$
\rho: B_{2 \mathrm{~d}}(\epsilon) \times(-\pi, \pi) \rightarrow X, \quad(p, q, \theta) \mapsto r_{e^{i \theta}}(\mathfrak{e}(\varrho(p, q))) ;
$$

here $B_{2 \mathrm{~d}}(\epsilon) \subseteq \mathbb{R}^{2 \mathrm{~d}}$ is a ball of radius $\epsilon$ centered at the origin, $\varrho: B_{2 \mathrm{~d}}(\epsilon) \rightarrow M$ is a preferred local chart for $M$ centered at $m=: \pi(x)$, meaning that it trivializes the holomorphic and symplectic structures at $m$, and $\mathfrak{e}$ is a unitary local frame of $A^{*}$, given by the unitarization of a preferred local frame (complete definitions are in [8]). Finally, $r_{e^{i \theta}}: X \rightarrow X$ is the diffeomorphism induced by the circle action. It is in this kind of local coordinate that the scaling limits of Szegö kernels exhibit their universal nature (Theorem 3.1 of [8]). If $\rho$ is a system of Heisenberg local coordinates centered at $x$, and $p, q \in \mathbb{R}^{\mathrm{d}}, w=p+i q$, one poses

$$
x+w=: \rho((p, q), 0) .
$$

For any $\theta$, we have

$$
u_{k}(\rho(p, q, \theta))=e^{i k \theta} u_{k}(\rho(p, q, 0))=e^{i k \theta} u_{k}(x+w) .
$$

Given that a system of Heisenberg local coordinates induces a unitary isomorphism of $T_{m} M$ and $\mathbb{C}^{\mathrm{d}}$, with this understanding we can also consider the expression $x+w$ with $w \in T_{m} M$.

If $x \in S^{1} \cdot \Lambda$, there are only finitely many elements $h_{1}, \ldots, h_{N_{x}} \in S^{1}$ such that $x_{j}=: r_{h_{j}}(x) \in \Lambda$. Since $\Lambda$ is Legendrian, hence horizontal for the connection, for any $j$ we may naturally identify the tangent space $T_{x_{j}} \Lambda \subseteq T_{x_{j}} X$ with a subspace of $T_{\pi(x)} M$. With this in mind, if $w \in T_{\pi(x)} M$ we can write $w=w_{j}^{\|}+w_{j}^{\perp}$ for unique $w_{j}^{\|} \in T_{x_{j}} \Lambda$ and $w_{j}^{\perp} \in T_{x_{j}} \Lambda^{\perp}$; the latter denotes the orthocomplement of $T_{x_{j}} \Lambda$ in $T_{\pi(x)} M$ in the Riemannian metric of $M$. 
Finally, let $\operatorname{dens}_{\Lambda}^{(1 / 2)}$ be the Riemannian half-density on $\Lambda$ (for the induced metric); thus if $\lambda$ is a $\mathcal{C}^{\infty}$ half-density on $\Lambda$ we can write $\lambda=F_{\lambda} \cdot \operatorname{dens}_{\Lambda}^{(1 / 2)}$ for a unique $F_{\lambda} \in \mathcal{C}^{\infty}(\Lambda)$.

Theorem 1. Let $\Lambda \subseteq X$ be a compact Legendrian submanifold, and suppose $\lambda$ is a smooth half-density on it. For every $k=1,2, \ldots$, let $u_{k}=: P_{k}\left(\delta_{\Lambda, \lambda}\right)$. Suppose $x \in S^{1} \cdot \Lambda$, and choose a system of Heisenberg local coordinates for $X$ centered at $x$. Let $h_{1}, \ldots, h_{N_{x}} \in S^{1}$ be the finitely many elements such that $r_{h_{j}}(x) \in \Lambda$. Then:

1. Suppose $a>0$. Uniformly for $\min _{j}\left\{\left\|w_{j}^{\perp}\right\|\right\} \gtrsim k^{a}$, we have

$$
u_{k}\left(x+\frac{w}{\sqrt{k}}\right)=O\left(k^{-\infty}\right) .
$$

2. There exist polynomials $a_{l j}$ on $\mathbb{C}^{d}$ such that the following holds: for $w \in$ $T_{\pi(x)} M$ and $k, \ell=1,2, \ldots$, let us define

$$
\begin{aligned}
R_{k, \ell}(x, w)=: & u_{k}\left(x+\frac{w}{\sqrt{k}}\right) \\
& -\left(\frac{2 k}{\pi}\right)^{d / 2} \sum_{j=1}^{N_{x}} h_{j}^{-k} e^{-\left\|w_{j}^{\perp}\right\|^{2}-i \omega_{\pi(x)}\left(w_{j}^{\perp}, w_{j}^{\|}\right)} F_{\lambda}\left(x_{j}\right) \\
& \cdot\left(1+\sum_{l=1}^{\ell} k^{-l / 2} a_{l j}(w)\right) .
\end{aligned}
$$

Then uniformly for $\|w\| \lesssim k^{1 / 6}$ we have

$$
\left|R_{k, \ell}(x, w)\right| \leq C_{\ell} k^{(d-\ell-1) / 2} \sum_{j=1}^{N_{x}} e^{-\frac{1-\epsilon}{2}\left\|w_{j}^{\perp}\right\|^{2}} .
$$

Corollary 1. $\forall w \in T_{\pi(x)} M$, the following asymptotic expansion holds as $k \rightarrow+\infty$ :

$$
\begin{aligned}
u_{k}\left(x+\frac{w}{\sqrt{k}}\right) \sim & \left(\frac{2 k}{\pi}\right)^{d / 2} \sum_{j=1}^{N_{x}} h_{j}^{-k} e^{-\left\|w_{j}^{\perp}\right\|^{2}-i \omega_{\pi(x)}\left(w_{j}^{\perp}, w_{j}^{\|}\right)} F_{\lambda}\left(x_{j}\right) \\
& \cdot\left(1+\sum_{l \geq 1} k^{-l / 2} a_{l j}(w)\right) .
\end{aligned}
$$

This agrees with Corollary 1.1 of 5 to leading order but gives a clearer picture of the asymptotic expansion, as well as an explicit estimate on the remainder.

The proof of Theorem 1 is based on the scaling asymptotics of Szegö kernels in Theorem 3.1 of $[8$, whereas the proofs in [5] are based on microlocal arguments that encompass the equivariant setting, involving a direct use of the parametrix developed in [3]. In view of the scaling asymptotics of equivariant Szegö kernels proved in [7, factorizations akin to Theorem 1 also hold in the equivariant setting; we shall not discuss this here.

\section{Proof of Theorem 1}

Let us first prove item 2. Thus, we want to investigate the asymptotics of $u_{k}\left(x+\frac{w}{\sqrt{k}}\right)$ as $k \rightarrow+\infty$, assuming that $w \in T_{\pi(x)} M,\|w\| \leq C k^{1 / 6}$ for some fixed $C>0$. 
Let $\Pi_{k} \in \mathcal{C}^{\infty}(X \times X)$ be the Schartz kernel of $\mathrm{P}_{k}$; explicitly, if $\left\{s_{r}^{(k)}\right\}$ is an orthonormal basis of $\mathcal{H}(X)_{k}$, then

$$
\Pi_{k}\left(y, y^{\prime}\right)=\sum_{r} s_{r}^{(k)}(y) \cdot \overline{s_{r}^{(k)}\left(y^{\prime}\right)} \quad\left(y, y^{\prime} \in X\right) .
$$

Let dens $X_{X}$ and dens $\Lambda$ denote, respectively, the Riemannian density on $X$ and $\Lambda$. Then, in standard distributional shorthand, by definition of $\delta_{\Lambda, \lambda}$ for any $x^{\prime} \in X$ we have

$$
\begin{aligned}
u_{k}\left(x^{\prime}\right) & =\int_{X} \Pi_{k}\left(x^{\prime}, y\right) \delta_{\Lambda, \lambda}(y) \operatorname{dens}_{X}(y) \\
& =\left\langle\delta_{\Lambda, \lambda}, \Pi_{k}\left(x^{\prime}, \cdot\right)\right\rangle=\int_{\Lambda} \Pi_{k}\left(x^{\prime}, y\right) F_{\lambda}(y) \operatorname{dens}_{\Lambda}(y) .
\end{aligned}
$$

Let us write $\operatorname{dist}_{M}$ for the Riemannian distance function on $M$, pulled back to a smooth function on $X \times X$ by the projection $\pi \times \pi$. Let us set:

$$
\begin{aligned}
& V_{k}=:\left\{x^{\prime} \in X: \operatorname{dist}_{M}\left(x, x^{\prime}\right)<4 C k^{-1 / 3}\right\}, \\
& V_{k}^{\prime}=:\left\{x^{\prime} \in X: \operatorname{dist}_{M}\left(x, x^{\prime}\right)>3 C k^{-1 / 3}\right\} .
\end{aligned}
$$

If $y \in V_{k}^{\prime}$ and $\|w\| \leq C k^{1 / 6}$, then $\operatorname{dist}_{M}\left(x+\frac{w}{\sqrt{k}}, y\right) \geq C k^{-1 / 3}$ for $k \gg 0$; by the off-diagonal estimates on Szegö kernels in [4], therefore, $\Pi_{k}\left(x+\frac{w}{\sqrt{k}}, y\right)=O\left(k^{-\infty}\right)$ uniformly for $y \in V_{k}^{\prime}$.

For $k \gg 0, \Lambda \cap V_{k}$ has $N_{x}$ connected components:

$$
\Lambda \cap V_{k}=\bigcup_{j=1}^{N_{x}} \Lambda_{k j},
$$

where $\Lambda_{k j}$ is the connected component containing $x_{j}$. Let $\left\{s_{k}, s_{k}^{\prime}\right\}$ be an $S^{1}$ invariant partition of unity on $X$ subordinate to the open cover $\left\{V_{k}, V_{k}^{\prime}\right\}$. In view of (2) and the previous discussion, we obtain

$$
\begin{aligned}
u_{k}\left(x+\frac{w}{\sqrt{k}}\right) & =\int_{\Lambda} \Pi_{k}\left(x+\frac{w}{\sqrt{k}}, y\right) F_{\lambda}(y) \operatorname{dens}_{\Lambda}(y) \\
& \sim \sum_{j=1}^{N_{x}} \int_{\Lambda_{k j}} \Pi_{k}\left(x+\frac{w}{\sqrt{k}}, y\right) F_{\lambda}(y) s_{k}(y) \operatorname{dens}_{\Lambda}(y),
\end{aligned}
$$

where $\sim$ means that the two terms have the same asymptotics. Let us now evaluate the asymptotics of the $j$-th summand in (3).

To this end, recall that the Heisenberg local chart $\rho$ centered at $x$ depends on the choice of the preferred local chart $\varrho$ at $\pi(x)$ and of the local frame $\mathfrak{e}$ of $A^{*}$. We obtain a Heisenberg local chart $\rho_{j}^{\prime}$ centered at $x_{j}$ by setting $\rho_{j}^{\prime}(p, q, \theta)=: r_{h_{j}}(\rho(p, q, \theta))$. By the discussion in $\S 2$ of [5] and (48) of the same paper, we can compose $\rho_{j}^{\prime}$ with a suitable transformation in $(p, q)$ (that is, a change of preferred local chart for $M)$ so as to obtain a Heisenberg local chart $\rho_{j}(p, q, \theta)$ centered at $x_{j}$ with the following property: $\Lambda$ is locally defined near $x_{j}$ by the conditions $\theta=f_{j}(q)$ and $p=0$, where $f_{j}$ vanishes to third order at the origin. By construction, we have $\rho_{j}(p, q, \theta)=r_{h_{j}}\left(\rho\left(p^{\prime}, q^{\prime}, \theta\right)\right)$ for a certain local diffeomorphism $(p, q) \mapsto\left(p^{\prime}, q^{\prime}\right)$. 
Thus $\Lambda$ is locally parametrized, near $x_{j}$ and in the chart $\rho_{j}$, by the imaginary vectors $i q$; viewing the $q$ 's as local coordinates on $\Lambda$ near $x_{j}$, we have dens $s_{\Lambda}=$ $D_{\lambda} \cdot|d q|$ for a unique locally defined smooth function $D_{\Lambda}$. By construction of Heisenberg local coordinates, $D_{\lambda}(0)=1$.

Applying a rescaling by $k^{-1 / 2}$, we obtain

$$
\begin{aligned}
& \int_{\Lambda_{k j}} \Pi_{k}\left(x+\frac{w}{\sqrt{k}}, y\right) F_{\lambda}(y) s_{k}(y) \operatorname{dens}_{\Lambda}(y) \\
& =k^{-\mathrm{d} / 2} \int_{\mathbb{R}^{\mathrm{d}}} \Pi_{k}\left(x+\frac{w}{\sqrt{k}}, r_{e^{i f_{j}(q / \sqrt{k})}}\left(x_{j}+\frac{i q}{\sqrt{k}}\right)\right) \\
& \cdot F_{\lambda}\left(\frac{q}{\sqrt{k}}\right) s_{k}\left(\frac{i q}{\sqrt{k}}\right) D_{\lambda}\left(\frac{q}{\sqrt{k}}\right) d q \\
& =k^{-\mathrm{d} / 2} \int_{\mathbb{R}^{\mathrm{d}}} e^{-i k f_{j}\left(\frac{q}{\sqrt{k}}\right)} \Pi_{k}\left(x+\frac{w}{\sqrt{k}}, x_{j}+\frac{i q}{\sqrt{k}}\right) \\
& \cdot F_{\lambda}\left(\frac{q}{\sqrt{k}}\right) s_{k}\left(\frac{i q}{\sqrt{k}}\right) D_{\lambda}\left(\frac{q}{\sqrt{k}}\right) d q .
\end{aligned}
$$

Here, $x+\frac{w}{\sqrt{k}}=\rho\left(\frac{\Re(w)}{\sqrt{k}}, \frac{\Im(w)}{\sqrt{k}}, 0\right)$ (we use the Heisenberg chart to unitarily identify $T_{m} M$ with $\left.\mathbb{C}^{\mathrm{d}}\right)$ and $x_{j}+\frac{i q}{\sqrt{k}}=\rho_{j}\left(0, \frac{q}{\sqrt{k}}, 0\right)$. Notice that $s_{k}\left(\frac{i q}{\sqrt{k}}\right)=1$ for $\|q\| \lesssim k^{1 / 6}$ and $s_{k}\left(\frac{i q}{\sqrt{k}}\right)=0$ for $\|q\| \gtrsim k^{1 / 6}$. In particular, integration takes place over a ball of radius $\sim k^{1 / 6}$. Also, Taylor expanding $F_{\lambda}$ and $f_{j}$ at the origin, we have asymptotic expansions

$$
F_{\lambda}\left(\frac{q}{\sqrt{k}}\right) \sim F_{\lambda}\left(x_{j}\right)+\sum_{r \geq 1} k^{-r / 2} b_{r}(q), \quad D_{\lambda}\left(\frac{q}{\sqrt{k}}\right) \sim 1+\sum_{r \geq 1} k^{-r / 2} c_{r}(q),
$$

and, since $f_{j}$ vanishes to third order at the origin,

$$
f_{j}\left(\frac{q}{\sqrt{k}}\right) \sim \sum_{r \geq 0} k^{-(3+r) / 2} d_{r}(q), \quad e^{-i k f_{j}\left(\frac{q}{\sqrt{k}}\right)} \sim 1+\sum_{r \geq 1} k^{-r / 2} e_{r}(q),
$$

for suitable polynomials $b_{r}, c_{r}, d_{r}, e_{r}$.

Let $w_{j} \in \mathbb{C}^{\mathrm{d}}$ correspond to $w$ in the Heisenberg local coordinates $\rho_{j}$. By the above, Taylor expanding the transformation $(p, q) \mapsto\left(p^{\prime}, q^{\prime}\right)$, we obtain $x+\frac{w}{\sqrt{k}}=$ $r_{h_{j}^{-1}}\left(x_{j}+\frac{w_{j}}{\sqrt{k}}+H(w, k)\right)$, where $H(w, k) \sim \sum_{f \geq 2} k^{-f / 2} h_{f}(w)$. Without affecting the leading order term of the resulting asymptotic expansion, we may pretend for simplicity that $x+\frac{w}{\sqrt{k}}=r_{h_{j}^{-1}}\left(x_{j}+\frac{w_{j}}{\sqrt{k}}\right)$.

Write $w_{j}=p_{j}+i q_{j}$, with $p_{j}, q_{j} \in \mathbb{R}^{\mathrm{d}}$. Thus $w_{j}^{\perp}=p_{j}, w_{j}^{\|}=i q_{j}$. In view of Theorem 3.1 of [8], we have

$$
\begin{aligned}
& \Pi_{k}\left(x+\frac{w}{\sqrt{k}}, x_{j}+\frac{i q}{\sqrt{k}}\right) \\
& =\Pi_{k}\left(r_{h_{j}^{-1}}\left(x_{j}+\frac{w_{j}}{\sqrt{k}}\right), x_{j}+\frac{i q}{\sqrt{k}}\right)=h_{j}^{-k} \Pi_{k}\left(x_{j}+\frac{w_{j}}{\sqrt{k}}, x_{j}+\frac{i q}{\sqrt{k}}\right) \\
& \sim h_{j}^{-k}\left(\frac{k}{\pi}\right)^{\mathrm{d}} e^{-i p_{j} \cdot q-\frac{1}{2}\left\|p_{j}\right\|^{2}-\frac{1}{2}\left\|q_{j}-q\right\|^{2}} \cdot\left(1+\sum_{r \geq 1} k^{-r / 2} R_{j}(w, q)\right),
\end{aligned}
$$


for certain polynomials $R_{j}$ in $w$ and $q$. Furthermore, by the large ball estimate on the remainder discussed in $\S 5$ of [8], the remainder after summing over $1 \leq r \leq R$ is bounded by

$$
C_{R} k^{\mathrm{d}-(R+1)} e^{-\frac{1-\epsilon}{2}\left(\left\|p_{j}\right\|^{2}+\left\|q-q_{j}\right\|^{2}\right)} .
$$

It follows that the product of these asymptotic expansions can be integrated term by term; given this, we only lose a contribution which is $O\left(k^{-\infty}\right)$ by setting $s_{k}=1$ and integrating over all of $\mathbb{R}^{\mathrm{d}}$.

We have

$$
\int_{\mathbb{R}^{\mathrm{d}}} e^{-i p_{j} \cdot q-\frac{1}{2}\left\|q_{j}-q\right\|^{2}} d q=e^{-i p_{j} \cdot q_{j}} \int_{\mathbb{R}^{\mathrm{d}}} e^{-i p_{j} \cdot s-\frac{1}{2}\|s\|^{2}} d s=(2 \pi)^{\mathrm{d} / 2} e^{-i p_{j} \cdot q_{j}-\frac{1}{2}\left\|p_{j}\right\|^{2}} .
$$

Given (5), this implies that (4) is given by an asymptotic expansion with leading order term

$$
h_{j}^{-k}\left(\frac{2 k}{\pi}\right)^{\mathrm{d} / 2} e^{-\left\|w_{j}^{\perp}\right\|^{2}-i \omega_{\pi(x)}\left(w_{j}^{\perp}, w_{j}^{\|}\right)} F_{\lambda}\left(x_{j}\right) .
$$

To determine the general term of the expansion, on the other hand, we are led to computing integrals of the form

$$
\int_{\mathbb{R}^{\mathrm{d}}} q^{\beta} e^{-i p_{j} \cdot q-\frac{1}{2}\left\|q_{j}-q\right\|^{2}} d q=e^{-i p_{j} \cdot q_{j}} \int_{\mathbb{R}^{\mathrm{d}}}\left(s+q_{j}\right)^{\beta} e^{-i p_{j} \cdot s-\frac{1}{2}\|s\|^{2}} d s,
$$

where $q^{\beta}$ is some monomial. Thus we are led to a sum of terms of the form

$$
e^{-i p_{j} \cdot q_{j}} C_{\gamma}\left(q_{j}\right) \int_{\mathbb{R}^{\mathrm{d}}} s^{\gamma} e^{-i p_{j} \cdot s-\frac{1}{2}\|s\|^{2}} d s,
$$

and the integral is the evaluation at $p_{j}$ of the Fourier transform of $s^{\gamma} e^{-\frac{1}{2}\|s\|^{2}}$. Up to a scalar factor, the latter is an iterated derivative to $e^{-\frac{1}{2}\|s\|^{2}}$; therefore we are left with a summand of the form $e^{-i p_{j} \cdot q_{j}} T\left(q_{j}, q_{j}\right) e^{-\frac{1}{2}\left\|p_{j}\right\|^{2}} d s$, where $T$ is a polynomial in $p_{j}, q_{j}$. Given (5), this implies that the general term of the asymptotic expansion for (4) has the form

$$
h_{j}^{-k}\left(\frac{2 k}{\pi}\right)^{\mathrm{d} / 2} k^{-l / 2} e^{-\left\|w_{j}^{\perp}\right\|^{2}-i \omega_{\pi(x)}\left(w_{j}^{\perp}, w_{j}^{\|}\right)} F_{\lambda}\left(x_{j}\right) \cdot a_{l j}(w)
$$

for an appropriate polynomial $a_{l j}(w)$. Finally, $(1)\left(\right.$ at $\left.x_{j}\right)$ follows by integrating (6).

To complete the proof of item 2, we need only sum over $j$.

Turning to the proof of item 1, by definition of preferred local coordinates, if $w_{j}^{\perp} \geq C k^{a}$, say, then

$$
\operatorname{dist}_{M}\left(x+\frac{v}{\sqrt{k}}, \Lambda_{k j}\right) \geq \frac{C}{2} k^{a-\frac{1}{2}}
$$

for all $k \gg 0$. By the off-diagonal estimates of [4], $\Pi_{k}\left(x+\frac{w}{\sqrt{k}}, y\right)=O\left(k^{-\infty}\right)$ uniformly for $y \in \Lambda_{k j}$. 


\section{REFERENCES}

[1] S. Bates, A. Weinstein, Lectures on the geometry of quantization, Berkeley Mathematics Lecture Notes 8, Amer. Math. Soc., Providence, RI; Berkeley Center for Pure and Applied Math., Berkeley, CA, 1997. MR.1806388 (2002f:53151)

[2] D. Borthwick, T. Paul, A. Uribe, Legendrian distributions with applications to relative Poincaré series, Invent. Math. 122 (2) (1995), 359-402. MR1358981 (97a:58188)

[3] L. Boutet de Monvel, J. Sjöstrand, Sur la singularité des noyaux de Bergman et de Szegö, Astérisque 34-35 (1976), 123-164. MR0590106 (58:28684)

[4] M. Christ, Slow off-diagonal decay for Szegö kernels associated to smooth Hermitian line bundles, Harmonic analysis at Mount Holyoke (South Hadley, MA, 2001), Contemp. Math., vol. 320, Amer. Math. Soc., Providence, RI, 2003, pp. 77-89. MR1979933 (2005b:32038)

[5] M. Debernardi, R. Paoletti, Equivariant asymptotics for Bohr-Sommerfeld Lagrangian submanifolds, Comm. Math. Phys. 267 (1) (2006), 227-263. MR2238910(2007g:58028)

[6] V. Guillemin, S. Sternberg, The Gelfand-Cetlin system and quantization of the complex flag manifold, J. Func. Anal. 52 (1983), 106-128. MR705993(85e:58069)

[7] R. Paoletti, Scaling limits for equivariant Szegö kernels, J. Symplectic Geom., to appear.

[8] B. Shiffman, S. Zelditch, Asymptotics of almost holomorphic sections of ample line bundles on symplectic manifolds, J. Reine Angew. Math. 544 (2002), 181-222. MR1887895(2002m:58043)

[9] A. Weinstein, Symplectic geometry, Bull. Amer. Math. Soc. (N.S.) 5 (1) (1981), 1-13. MR614310 (83a:58044)

Dipartimento di Matematica e Applicazioni, Università degli Studi di Milano Bicocca, Via R. Cozzi 53, 20125 Milano, Italy

E-mail address: roberto.paoletti@unimib.it 